\title{
EXISTENCE OF COMPATIBLE CONTACT STRUCTURES ON $G_{2}$-MANIFOLDS*
}

\author{
M. FIRAT ARIKAN ${ }^{\dagger}$, HYUNJOO CHO AND SEMA SALUR $^{\S}$
}

\begin{abstract}
In this paper, we show the existence of (co-oriented) contact structures on certain classes of $G_{2}$-manifolds, and that these two structures are compatible in certain ways. Moreover, we prove that any seven-manifold with a spin structure (and so any manifold with $G_{2}$-structure) admits an almost contact structure. We also construct explicit almost contact metric structures on manifolds with $G_{2}$-structures.
\end{abstract}

Key words. (Almost) contact structures, $G_{2}$ structures.

AMS subject classifications. 53C38, 53D10, 53D15, 57R17.

1. Introduction. Let $(M, g)$ be a Riemannian 7-manifold whose holonomy group $\operatorname{Hol}(g)$ is the exceptional Lie group $G_{2}$ (or, more generally, a subgroup of $G_{2}$ ). Then $M$ is naturally equipped with a covariantly constant 3 -form $\varphi$ and 4 -form $* \varphi$. We can define $(M, \varphi, g)$ as the $G_{2}$-manifold with $G_{2}$ structure $\varphi$.

We can also define a (co-oriented) contact manifold as a pair $(N, \xi)$ where $N$ is an odd-dimensional manifold and $\xi$, called a (co-oriented) contact structure, is a totally non-integrable (co-oriented) hyperplane distribution on $N$.

In dimension 7 , so far contact geometry and $G_{2}$ geometry have been studied independently and each geometry has very distinguished characteristics which are rather different than those in the other. A basic example of such differences is the following: In contact geometry there are no local invariants, in other words, every contact 7-manifold is locally contactomorphic to $\mathbb{R}^{7}$ equipped with the standard contact structure. On the other hand, in $G_{2}$ geometry it is the $G_{2}$ structure itself that determines how local neighborhoods of points look like, and as a result, manifolds with $G_{2}$ structures can look the same only at a point, [7], [9].

The aim of this paper is to initiate a new interdisciplinary research area between contact and $G_{2}$ geometries. More precisely, we study the existence of (almost) contact structures on 7-dimensional manifolds with (torsion-free) $G_{2}$-structures.

The paper is organized as follows: After the preliminaries (Section 2), we show the existence of almost contact structures on 7 -manifolds with spin structures in Section 3. In particular, we prove the following theorem:

THEOREM. Every manifold with $G_{2}$-structure admits an almost contact structure.

In Section 4 , we define $A$ - and $B$-compatibility between contact and $G_{2}$ structures, and also present the motivating example for $\mathbb{R}^{7}$. We also prove the nonexistence result:

Theorem. Let $(M, \varphi)$ be a manifold with $G_{2}$-structure such that $d \varphi=0$. If $M$ is closed (i.e., compact and $\partial M=\emptyset$ ), then there is no contact structure on $M$ which

\footnotetext{
*Received February 9, 2012; accepted for publication April 3, 2012.

$\dagger$ Department of Mathematics, University of Rochester, Rochester NY, USA; Max Planck Institute for Mathematics, Bonn, Germany (arikan@math.rochester.edu; arikan@mpim-bonn.mpg.de). The first named author is partially supported by NSF FRG grant DMS-1065910.

${ }^{\ddagger}$ Department of Mathematics, University of Rochester, Rochester NY, USA (cho@math.rochester. $\mathrm{edu})$.

$\S$ Department of Mathematics, University of Rochester, Rochester NY, USA (salur@math. rochester.edu). The third named author is partially supported by NSF grant DMS-1105663.
} 
is A-compatible with $\varphi$.

In Section 5, for any non-vanishing vector field $R$ on a manifold $M$ with $G_{2}$-structure $\varphi$, we construct explicit almost contact structure, denoted by $\left(J_{R}, R, \alpha_{R}, g_{\varphi}\right)$, and indeed prove the following theorems:

ThEOREM. Let $(M, \varphi)$ be a manifold with $G_{2}$-structure. Then the quadruple $\left(J_{R}, R, \alpha_{R}, g_{\varphi}\right)$ defines an almost contact metric structure on $M$ for any non-vanishing vector field $R$ on $M$. Moreover, such a structure exists on any manifold with $G_{2}$ structure.

Theorem. Let $(M, \varphi)$ be a manifold with $G_{2}$-structure. Suppose that $\xi$ is a contact structure on $M$ such that $\left(J_{R}, R, \alpha_{R}, g_{\varphi}\right)$ is an associated almost contact metric structure for $\xi$. Then $\xi$ is A-compatible.

In Section 6, we define contact- $G_{2}$-structures on 7 -manifolds and analyze their relations with $A$-compatible contact structures, the main results of that section are:

ThEOREM. Let $(M, \varphi)$ be a manifold with $G_{2}$-structure. Assume that there are nowhere-zero vector fields $X, Y$ and $Z$ on $M$ satisfying $\iota_{Z} \varphi=Y^{b} \wedge X^{b}$ where $X^{b}$ (resp. $\left.Y^{b}\right)$ is the covariant 1 -form of $X$ (resp. $Y$ ) with respect to the $G_{2}$-metric $g_{\varphi}$. Also suppose that $d\left(i_{X} i_{Y} \varphi\right)=i_{X} i_{Y} * \varphi$. Then the 1 -form $\alpha:=Z^{b}=g_{\varphi}(Z, \cdot)$ is a contact form on $M$ and it defines an A-compatible contact structure $\operatorname{Ker}(\alpha)$ on $(M, \varphi)$.

THEOREM. Let $(\varphi, R, \alpha, f, g)$ be a contact-G $G_{2}$-structure on a smooth manifold $M^{7}$. Then $\alpha$ is a contact form on $M$. Moreover, $\xi=\operatorname{Ker}(\alpha)$ is an A-compatible contact structure on $(M, \varphi)$. In particular, if $M$ is closed, then it does not admit a contact- $G_{2}-$ structure with $d \varphi=0$.

Theorem. Let $(M, \varphi)$ be any manifold with $G_{2}$-structure. Then every $A$ compatible contact structure on $(M, \varphi)$ determines a contact-G $G_{2}-$ structure on $M$.

Finally, in Section 7, we present some examples of $A$-compatible structures and contact $-G_{2}-$ structures.

\section{Preliminaries.}

2.1. $G_{2}$-structures and $G_{2}$-manifolds. A smooth 7-dimensional manifold $M$ has a $G_{2}$-structure, if the structure group of $T M$ can be reduced to $G_{2}$. The group $G_{2}$ is one of the five exceptional Lie groups which is the group of all linear automorphisms of the imaginary octonions $i m \mathbb{O} \cong \mathbb{R}^{7}$ preserving a certain cross product. Equivalently, it can be defined as the subgroup of $G L(7, \mathbb{R})$ which preserves the 3 -form

$$
\varphi_{0}=e^{123}+e^{145}+e^{167}+e^{246}-e^{257}-e^{347}-e^{356}
$$

where $\left(x_{1}, \ldots, x_{7}\right)$ are the coordinates on $\mathbb{R}^{7}$, and $e^{i j k}=d x^{i} \wedge d x^{j} \wedge d x^{k}$. As an equivalent definition, a manifold with a $G_{2}$-structure $\varphi$ is a pair $(M, \varphi)$, where $\varphi$ is a 3 -form on $M$, such that $\left(T_{p} M, \varphi\right)$ is isomorphic to $\left(\mathbb{R}^{7}, \varphi_{0}\right)$ at every point $p$ in $M$. Such a $\varphi$ defines a Riemannian metric $g_{\varphi}$ on $M$. We say $\varphi$ is torsion-free if $\nabla \varphi=0$ where $\nabla$ is the Levi-Civita connection of $g_{\varphi}$. A Riemannian manifold with a torsion-free $G_{2}$-structure is called a $G_{2}$-manifold. Equivalently, the pair $(M, \varphi)$ is called a $G_{2}$-manifold if its holonomy group (with respect to $g_{\varphi}$ ) is a subgroup of $G_{2}$. As an another characterization, one can show that $\varphi$ is torsion-free if and only if $d \varphi=d(* \varphi)=0$ where "*" is the Hodge star operator defined by the metric $g_{\varphi}$. 
The 3 -form $\varphi$ also determines the cross product and the orientation top (volume) form $\operatorname{Vol}$ on $M$. In fact, for any vector fields $u, v, w$ on $M$, we have

$$
\begin{gathered}
\varphi(u, v, w)=g_{\varphi}(u \times v, w), \\
\left(\iota_{u} \varphi\right) \wedge\left(\iota_{v} \varphi\right) \wedge \varphi=6 g_{\varphi}(u, v) \text { Vol. }
\end{gathered}
$$

Also we will make use of the following formula as well:

$$
u \times(u \times v)=-\|u\|^{2} v+g_{\varphi}(u, v) u .
$$

See [2], [3], [9] and [10] for more details on $G_{2}$ geometry.

2.2. Contact and almost contact structures. A contact structure on a smooth $(2 n+1)$-dimensional manifold $M$ is a global $2 n$-plane field distribution $\xi$ which is totally non-integrable. Non-integrability condition is equivalent to the fact that locally $\xi$ can be given as the kernel of a 1 -form $\alpha$ such that $\alpha \wedge(d \alpha)^{n} \neq 0$. If $\alpha$ is globally defined (in such a case, it is called a contact form), then one can define the Reeb vector field of $\alpha$ to be the unique global nowhere-zero vector field $R$ on $M$ satisfying the equations

$$
\iota_{R} d \alpha=0, \quad \alpha(R)=1
$$

where " $\iota$ " denotes the interior product.

Using $R$, we can co-orient $\xi$ and, as a result, the structure group of the tangent frame bundle can be reduced to $U(n) \times 1$. Such a reduction of the structure group is called an almost contact structure on $M$. Therefore, for the existence of a cooriented contact structure on $M$, one should first ask the existence of an almost contact structure. We refer the reader to [1] and [7] for more on contact geometry.

Definition $2.1([8])$. Let $M^{2 n+1}$ be a smooth manifold. If the structure group of its tangent bundles $T M^{2 n+1}$ reduces to $U(n) \times 1$, then $M^{2 n+1}$ is said to have an almost contact structure.

3. Almost contact structures on 7-manifolds with a spin structure. Although no explicit description is given, nevertheless the following result shows the existence of almost contact structures not only on manifolds with $G_{2}$-structures but also on a much wider family of 7 -manifolds. Recall that if a manifold admits a spin structure, then its second Stiefel-Whitney class is zero.

THEOREM 3.1. Every 7-manifold with a spin structure admits an almost contact structure.

Proof. Assume that $M$ is a 7 -manifold with a spin structure. By definition, $M$ admits an almost contact structure if and only if the structure group of $T M$ can be reduced to $U(3) \times 1$. Equivalently, the associated fiber bundle $T M[S O(7) / U(3)]$ with fiber $S O(7) / U(3)$ admits a cross-section [13]. If $s$ is a cross section of fiber bundle over the the $(i-1)$-skeleton of $M$, then the cohomology class

$$
o^{i}(s) \in H^{i}\left(M, \pi_{i-1}(S O(7) / U(3))\right.
$$


is the obstruction to extending $s$ over the $i$-skeleton. Since we have

$$
\pi_{i}(S O(7) / U(3))=0
$$

unless $i=2,6$, the only obstructions to the existence of such a cross section arise in $H^{i}(M, \mathbb{Z})$ for $i=3,7$. In [11], Massey shows that these obstructions are the integral Stiefel-Whitney classes of the associated dimensions. Recall that the integral StiefelWhitney classes are defined as the images $\beta\left(w_{i}\right)$ of the Stiefel-Whitney classes under the Bockstein homomorphism. Here the Bockstein homomorphism is the connecting homomorphism $\beta: H^{i}(M, \mathbb{Z} / 2 \mathbb{Z}) \rightarrow H^{i+1}(M, \mathbb{Z})$ which arises from the short exact sequence

$$
0 \longrightarrow \mathbb{Z} \stackrel{\times 2}{\longrightarrow} \mathbb{Z} \longrightarrow \mathbb{Z} / 2 \mathbb{Z} \longrightarrow 0 .
$$

Therefore, the obstructions $o^{3}, o^{7}$ to the existence of an almost contact structures on 7-manifolds are 2-torsion classes.

Now we know that $w_{2}(M)=0$ (since $M$ is spin), and hence the third integral Stiefel-Whitney class vanishes, i.e., $o^{3}=W_{3}(M)=\beta\left(w_{2}\right)=0$. Therefore, the only obstruction lies in the cohomology group $H^{7}(M)$.

We consider the following cases: First, if $M$ is a closed manifold, then by Poincaré duality $H^{7}(M) \cong H_{0}(M) \cong \mathbb{Z}$ and hence the top-dimensional obstruction $o^{7}$ vanishes. Secondly, if $M$ has a boundary, then (again by the duality) we have $o^{7} \in H^{7}(M) \cong$ $H_{0}(M, \partial M) \cong 0$. Now, if $M$ is non-compact without a boundary, then the cohomology group $H^{7}(M) \cong\left(H_{c s}^{0}(M)\right)^{*}$ where $H_{c s}$ denotes the compactly supported cohomology. Hence, it is torsion-free.

Since every manifold with $G_{2}$-structure is spin, we get

COROllary 3.2. Every manifold with $G_{2}$-structure admits an almost contact structure.

4. Compatibility and the motivating example. Assuming the existence of a contact structure on a manifold with a $G_{2}$-structure, we can also ask if and how these two structures are related. We define two different notions of compatibility between them as follows:

Definition 4.1. A (co-oriented) contact structure $\xi$ on $(M, \varphi)$ is said to be $A$ compatible with the $G_{2}$-structure $\varphi$ if there exist a vector field $R$ on $M$ and a nonzero function $f: M \rightarrow \mathbb{R}$ such that $d \alpha=\iota_{R} \varphi$ for some contact form $\alpha$ for $\xi$ and $f R$ is the Reeb vector field of a contact form for $\xi$.

Definition 4.2. A (co-oriented) contact structure $\xi$ on $(M, \varphi)$ is said to be $B$-compatible with the $G_{2}$-structure $\varphi$ if there are (global) vector fields $X, Y$ on $M$ such that $\alpha=\iota_{Y} \iota_{X} \varphi$ is a contact form for $\xi$.

In this paper, we will mainly consider $A$-compatible contact structures. We remark that if $\varphi$ is torsion-free or at least $d \varphi=0$, then Definition 4.1 makes sense only if $M$ is noncompact or compact with boundary. Indeed, we can easily prove the following:

THEOREM 4.3. Let $(M, \varphi)$ be a manifold with $G_{2}$-structure such that $d \varphi=0$. If $M$ is closed (i.e., compact and $\partial M=\emptyset$ ), then there is no contact structure on $M$ which is A-compatible with $\varphi$. 
Proof. Suppose $\xi$ is an A-compatible contact structure on $(M, \varphi)$. Therefore, $d \alpha=\iota_{R} \varphi$ for some contact form $\alpha$ for $\xi$ and some nonvanishing vector field $R$. Using the equation (2), we have

$$
d \alpha \wedge d \alpha \wedge \varphi=\left(\iota_{R} \varphi\right) \wedge\left(\iota_{R} \varphi\right) \wedge \varphi=6\|R\|^{2} \text { Vol. }
$$

Since $d \varphi=0$, we have $d \alpha \wedge d \alpha \wedge \varphi=d(\alpha \wedge d \alpha \wedge \varphi)$. Now by Stokes' Theorem,

$$
0 \supsetneqq \int_{M} 6\|R\|^{2} \mathrm{Vol}=\int_{M} d(\alpha \wedge d \alpha \wedge \varphi)=\int_{\partial M} \alpha \wedge d \alpha \wedge \varphi=0
$$

(as $\partial M=\emptyset$ ). This gives a contradiction.

For another application of this argument on specific vector fields on manifolds with $G_{2}$ structures, see [5].

We now explore the relation between the standard contact structure $\xi_{0}$ and the standard $G_{2}$-structure $\varphi_{0}$ on $\mathbb{R}^{7}$. Indeed, the notion of A- and B-compatibility relies on this motivating example.

Fix the coordinates $\left(x_{1}, x_{2}, x_{3}, x_{4}, x_{5}, x_{6}, x_{7}\right)$ on $\mathbb{R}^{7}$. In these coordinates,

$$
\varphi_{0}=e^{123}+e^{145}+e^{167}+e^{246}-e^{257}-e^{347}-e^{356}
$$

where $e^{i j k}$ denotes the 3 -form $d x_{i} \wedge d x_{j} \wedge d x_{k}$. Consider the standard contact structure $\xi_{0}$ on $\mathbb{R}^{7}$ as the kernel of the 1 -form

$$
\alpha_{0}=d x_{1}-x_{3} d x_{2}-x_{5} d x_{4}-x_{7} d x_{6} .
$$

For simplicity, through out the paper we will denote $\partial / \partial x_{i}$ by $\partial x_{i}$ (so we have $\left.d x_{i}\left(\partial x_{j}\right)=\delta_{i j}\right)$. Consider the vector fields

$$
R=\partial x_{1}, X=\partial x_{7} \text { and } Y=-x_{7} \partial x_{1}+x_{5} \partial x_{3}-x_{3} \partial x_{5}-\partial x_{6}+f \partial x_{7}
$$

where $f: \mathbb{R}^{7} \rightarrow \mathbb{R}$ is any smooth function (in fact, it is enough to take $f \equiv 0$ for our purpose). By a straightforward computation, we see that

$$
d \alpha_{0}=\iota_{R}\left(\varphi_{0}\right), \quad \alpha_{0}=\iota_{Y} \iota_{X}\left(\varphi_{0}\right)
$$

Also observe that $R$ is the Reeb vector field of $\alpha_{0}$. Note that this contact structure is not unique A-compatible with $\varphi_{0}$. In fact we have various ways of choosing the contact structures by rotating indexes and signes. For example, the contact structure $\alpha=$ $d x_{2}+x_{3} d x_{1}-x_{6} d x_{4}+x_{7} d x_{5}$ with $R=\partial x_{2}$ is another A-compatible contact structure with $\varphi_{0}$ and by choosing two vectors $X=\partial x_{7}, Y=\partial x_{5}-x_{3} \partial x_{6}+x_{6} \partial x_{3}-x_{7} \partial x_{2}+f \partial x_{7}$ it is easily seen as being B-compatible with $\varphi_{0}$. Therefore, we have proved:

THEOREM 4.4. There are contact structures $\xi$ on $\mathbb{R}^{7}$ which are both $A$ - and $B$-compatible with the standard $G_{2}$-structure $\varphi_{0}$.

5. An explicit almost contact metric structure. We first give an alternative definition of an almost contact structure, and then construct an explicit almost contact structure on a manifold with $G_{2}$-structure. The reader is referred to [1] for the equivalence between the previous definition (Definition 2.1) and this new one.

DEFINITION $5.1([12])$. An almost contact structure on a differentiable manifold $M^{2 n+1}$ is a triple $(J, R, \alpha)$ consists of a field $J$ of endomorphisms of the tangent spaces, a vector field $R$, and a 1 -form $\alpha$ satisfying 
(i) $\alpha(R)=1$,

(ii) $J^{2}=-I+\alpha \otimes R$

where $I$ denotes the identity transformation.

For completeness, we provide the proof of the following lemma.

LEMma $5.2([12])$. Suppose that $(J, R, \alpha)$ is an almost contact structure on $M^{2 n+1}$. Then $J(R)=0$ and $\alpha \circ J=0$

Proof. Since $J^{2}(R)=-R+\alpha(R) R=-R+1 \cdot R=0$, we have either $J(R)=0$ or $J(R)$ is nonzero vector field whose image is 0 . Suppose $J(R)$ is nonzero vector field which is mapped to 0 by $J$. Then from

$$
0=J^{2}(J(R))=-J(R)+\alpha(J(R)) \cdot R
$$

we get $J(R)=\alpha(J(R)) \cdot R$, and so $\alpha(J(R)) \neq 0($ as $J(R) \neq 0)$. But then

$$
J^{2}(R)=J(J(R))=J(\alpha(J(R)) R)=\alpha(J(R)) \cdot J(R)=[\alpha(J(R))]^{2} \cdot R \neq 0
$$

which contradicts to assumption that $J^{2}(R)=J(J(R))=0$. Hence, we conclude that $J(R)=0$ must be the case.

Now for any vector $X$, we see that

$$
J^{3}(X)=J\left(J^{2}(X)\right)=J((-X)+\alpha(X) R)=-J(X)+J(\alpha(X) R)
$$

and also we have

$$
J^{3}(X)=J^{2}(J(X))=-J(X)+\alpha(J(X)) R
$$

So combining these we compute

$$
\begin{aligned}
\alpha(J(X)) R & =J^{3}(X)+J(X) \\
& =-J(X)+J(\alpha(X) R)+J(X)=J(\alpha(X) R) .
\end{aligned}
$$

But using the fact $J(R)=0$ we have

$$
J(\alpha(X) R)=\alpha(X) J(R)=0 .
$$

Therefore, $\alpha(J(X))=0$ as $R \neq 0$. Hence, $\alpha \circ J=0$ for any vector $X$.

We can also introduce a Riemannian metric into the picture as suggested in the following definition.

DEFINITION $5.3([12])$. An almost contact metric structure on a differentiable manifold $M^{2 n+1}$ is a quadruple $(J, R, \alpha, g)$ where $(J, R, \alpha)$ is an almost contact structure on $M$ and $g$ is a Riemannian metric on $M$ satisfying

$$
g(J u, J v)=g(u, v)-\alpha(u) \alpha(v)
$$

for all vector fields $u, v$ in $T M$. Such a $g$ is called a compatible metric.

REMARK 5.4. Every manifold with an almost contact structure admits a compatible metric (see [1], for a proof). Also setting $u=R$ in Equation (5) gives 
$g(J R, J v)=g(R, v)-\alpha(R) \alpha(v)$. Since $J(R)=0$, an immediate consequence is that $\alpha$ is the covariant form of $R$, that is, $\alpha(v)=g(R, v)$.

Definition 5.5 ([12]). Let $M$ be an odd-dimensional manifold, and $\alpha$ be a contact form on $M$ with the Reeb vector field $R$. Therefore, $d \alpha$ is a symplectic form on the contact structure (or distribution) $\xi=\operatorname{Ker}(\alpha)$. We say that the triple $(J, R, \alpha)$ is an associated almost contact structure for $\xi$ if $J$ is $d \alpha$-compatible almost complex structure on the complex bundle $\xi$, that is

$$
d \alpha(J X, J Y)=d \alpha(X, Y) \text { and } d \alpha(X, J X)>0 \text { for all } X, Y \in \xi
$$

Furthermore, if $g$ is a metric on $M$, we consider two equations:

$$
\begin{gathered}
g(J X, J Y)=g(X, Y)-\alpha(X) \alpha(Y) \\
d \alpha(X, Y)=g(J X, Y)
\end{gathered}
$$

for all $X, Y \in T M$. We say that $(J, R, \alpha, g)$ is an associated almost contact metric structure if two equations (6) and (7) hold. In this case, $g$ is called an associated metric.

Suppose that $(M, \varphi)$ is a manifold with $G_{2}$-structure. There might be many ways to construct almost contact metric structures on $(M, \varphi)$. Here we give a particular way of constructing almost contact metric structures on $(M, \varphi)$. Denote the Riemannian metric and the cross product (determined by $\varphi$ ) by $g_{\varphi}=\langle\cdot, \cdot\rangle_{\varphi}$ and $\times_{\varphi}$, respectively. Suppose that $R$ is a nowhere vanishing vector field on $M$. By normalizing $R$ using $g_{\varphi}$, we may assume that $\|R\|=1$. Then using the metric, we define the 1 -form $\alpha_{R}$ as the metric dual of $R$, that is,

$$
\alpha_{R}(u)=g_{\varphi}(R, u)=\langle R, u\rangle_{\varphi} .
$$

Moreover, using the cross product and $R$, we can define an endomorphism $J_{R}: T M \rightarrow$ $T M$ of the tangent spaces by

$$
J_{R}(u)=R \times_{\varphi} u .
$$

Note that $J_{R}(R)=0$, and so $J_{R}$, indeed, defines a complex structure on the orthogonal complement $R^{\perp}$ of $R$ with respect to $g_{\varphi}$. With these, we have

THeOREM 5.6. Let $(M, \varphi)$ be a manifold with $G_{2}$-structure. Then the quadruple $\left(J_{R}, R, \alpha_{R}, g_{\varphi}\right)$ defines an almost contact metric structure on $M$ for any non-vanishing vector field $R$ on $M$. Moreover, such a structure exists on any manifold with $G_{2}$ structure.

Proof. As before, we will assume that $R$ is already normalized using $g_{\varphi}$. First, note that $\alpha_{R}(R)=g_{\varphi}(R, R)=\|R\|^{2}=1$. Also we have

$$
J_{R}^{2}(u)=J_{R}\left(R \times_{\varphi} u\right)=R \times_{\varphi}\left(R \times_{\varphi} u\right)=-\|R\|^{2} u+g_{\varphi}(R, u) R=-u+\alpha(u) R
$$

where we made use of the identity (3). This shows that the endomorphism $J_{R}$ : $T M \rightarrow T M$ satisfies the condition

$$
J_{R}^{2}=-I+\alpha \otimes R .
$$


Therefore, the triple $\left(J_{R}, R, \alpha_{R}\right)$ is an almost contact structure on $M$. Next, we check $g_{\varphi}$ is a compatible metric with this structure. Using (1) and (3), we compute

$$
\begin{aligned}
g_{\varphi}\left(J_{R} u, J_{R} v\right) & =g_{\varphi}\left(R \times_{\varphi} u, R \times_{\varphi} v\right)=\varphi\left(R, u, R \times_{\varphi} v\right)=-\varphi\left(R, R \times_{\varphi} v, u\right) \\
& =-g_{\varphi}\left(R \times_{\varphi}\left(R \times_{\varphi} v\right), u\right)=-g_{\varphi}\left(-\|R\|^{2} v+g_{\varphi}(R, v) R, u\right) \\
& =-g_{\varphi}\left(-v+g_{\varphi}(R, v) R, u\right)=g_{\varphi}(v, u)-g_{\varphi}\left(\alpha_{R}(v) R, u\right) \\
& ==g_{\varphi}(u, v)-\alpha_{R}(v) \underbrace{g_{\varphi}(R, u)}_{\alpha_{R}(u)}=g_{\varphi}(u, v)-\alpha_{R}(u) \alpha_{R}(v)
\end{aligned}
$$

which holds for all vector fields $u, v$ in $T M$. This proves that $g_{\varphi}$ satisfies (5). Hence, $\left(J_{R}, R, \alpha_{R}, g_{\varphi}\right)$ is an almost contact metric structure on $M$.

For the last statement, we know that there always exists a nowhere vanishing vector field $R$ on any 7-dimensional manifold. In particular, $\left(J_{R}, R, \alpha_{R}, g_{\varphi}\right)$ can be constructed on any manifold $M$ with $G_{2}$-structure $\varphi$.

THEOREM 5.7. Let $(M, \varphi)$ be a manifold with $G_{2}$-structure, and $\left(J_{R}, R, \alpha_{R}, g_{\varphi}\right)$ be an almost contact metric structure on $M$ constructed as above. Suppose that $\xi$ is a contact structure on $M$ such that $\left(J_{R}, R, \alpha_{R}, g_{\varphi}\right)$ is an associated almost contact metric structure for $\xi$. Then $\xi$ is A-compatible.

Proof. By assumption $\left(J_{R}, R, \alpha_{R}, g_{\varphi}\right)$ is an associated almost contact metric structure for $\xi$. Therefore, $g_{\varphi}$ is an associated metric and satisfies

$$
d \alpha_{R}(u, v)=g_{\varphi}\left(J_{R}(u), v\right) \text { for all } u, v \in T M .
$$

But then using the equation defining $J_{R}$ and (1), we obtain

$$
d \alpha_{R}(u, v)=g_{\varphi}\left(R \times_{\varphi} u, v\right)=\varphi(R, u, v)=i_{R} \varphi(u, v), \quad \forall u, v \in T M .
$$

Therefore, we have $d \alpha_{R}=i_{R} \varphi$. Also $R$ is the Reeb vector field of $\alpha_{R}$ by assumption. Hence, $\xi$ is $A$-compatible by definition.

Corollary 5.8. Let $(M, \varphi)$ be a manifold with $G_{2}$-structure such that $d \varphi=0$, and $\left(J_{R}, R, \alpha_{R}, g_{\varphi}\right)$ be an almost contact metric structure on $M$ constructed as above. If $M$ is closed, then there is no contact structure on $M$ whose associated almost contact metric structure is $\left(J_{R}, R, \alpha_{R}, g_{\varphi}\right)$.

Proof. On the contrary, suppose that $\xi=\operatorname{Ker}\left(\alpha_{R}\right)$ is a contact structure on a closed manifold $M$ equipped with a $G_{2}$-structure $\varphi$ and $d \varphi=0$, and also that $\left(J_{R}, R, \alpha_{R}, g_{\varphi}\right)$ is an associated almost contact metric structure. Then, by Theorem $5.7, \xi$ is A-compatible, but this contradicts to Theorem 4.3.

6. Contact $-G_{2}-$ structures on 7 -manifolds. Suppose that $(M, \varphi)$ is a manifold with $G_{2}$-structure. Let us recall the decomposition of the space $\Lambda^{2}$ of 2 -forms on $M$ obtained from $G_{2}$-representation and some other useful formulas which we will use. A good source for these is [2] and also [9]. According to irreducible $G_{2}$-representation, $\Lambda^{2}=\Lambda_{7}^{2} \oplus \Lambda_{14}^{2}$ where

$$
\begin{aligned}
\Lambda_{7}^{2} & =\left\{i_{v} \varphi ; v \in \Gamma(T M)\right\} \\
& =\left\{\beta \in \Lambda^{2} ; *(\varphi \wedge \beta)=-2 \beta\right\} \\
& =\left\{\beta \in \Lambda^{2} ; *(* \varphi \wedge(*(* \varphi \wedge \beta)))=3 \beta\right\} \\
\Lambda_{14}^{2} & =\left\{\beta \in \Lambda^{2} ; * \varphi \wedge \beta=0\right\} \\
& =\left\{\beta \in \Lambda^{2} ; *(\varphi \wedge \beta)=\beta\right\}
\end{aligned}
$$


Also on any Riemannian $n$-manifold, for any $k$-form $\alpha$ and a vector field $v$, the following equalities hold:

$$
\begin{gathered}
i_{v} * \alpha=(-1)^{k} *\left(v^{b} \wedge \alpha\right) \quad \text { and } \\
i_{v} \alpha=(-1)^{n k+n} *\left(v^{b} \wedge * \alpha\right) .
\end{gathered}
$$

As a last one we recall a very useful equality: For any $k$-form $\lambda$, and any $(n+1-k)$ form $\mu$ and any vector field $v$ on a smooth manifold of dimension $n$, we have

$$
\left(\iota_{v} \lambda\right) \wedge \mu=(-1)^{k+1} \lambda \wedge\left(\iota_{v} \mu\right) .
$$

Now we are ready to prove:

TheOREM 6.1. Let $(M, \varphi)$ be a manifold with $G_{2}$-structure. Assume that there are nowhere-zero vector fields $X, Y$ and $Z$ on $M$ satisfying

$$
\iota_{Z} \varphi=Y^{b} \wedge X^{b}
$$

where $X^{b}$ (resp. $\left.Y^{b}\right)$ is the covariant 1-form of $X$ (resp. $Y$ ) with respect to the $G_{2}$-metric $g_{\varphi}$. Also suppose that

$$
d\left(i_{X} i_{Y} \varphi\right)=i_{X} i_{Y} * \varphi
$$

Then the 1-form $\alpha:=Z^{b}=g_{\varphi}(Z, \cdot)$ is a contact form on $M$ and it defines an $A$ compatible contact structure $\operatorname{Ker}(\alpha)$ on $(M, \varphi)$.

Proof. From (8) we know that $\iota_{Z} \varphi$ is an element of $\Lambda_{7}^{2}$. Set $\iota_{Z} \varphi=\beta \in \Lambda_{7}^{2}$, and so we have $\iota_{Z} \varphi=\beta=Y^{b} \wedge X^{b}$ by (12). Also applying (9) twice gives

$$
i_{X} i_{Y} * \varphi=-i_{X}\left(*\left(Y^{b} \wedge \varphi\right)\right)=-*\left(X^{b} \wedge Y^{b} \wedge \varphi\right)=*\left(Y^{b} \wedge X^{b} \wedge \varphi\right)=*(\beta \wedge \varphi)
$$

from which we get

$$
i_{X} i_{Y} * \varphi=-2 \beta
$$

where we use the second line in (8). Moreover, by (10) followed by (9),

$$
i_{X} i_{Y} \varphi=i_{X}\left(*\left(Y^{b} \wedge * \varphi\right)\right)=-*\left(X^{b} \wedge Y^{b} \wedge * \varphi\right)=*(\beta \wedge * \varphi) .
$$

Now putting (14) and (15) into (13) gives us

$$
d *(\beta \wedge * \varphi)=-2 \beta=-2 \iota_{Z} \varphi .
$$

Recall the formula $\left(i_{v} \varphi\right) \wedge * \varphi=3 * v^{b}$ which is true for any vector field $v$. By taking $v=Z$, we compute the left-hand side in (16) as

$$
d *(\beta \wedge * \varphi)=d *\left(3 * Z^{b}\right)=3 d Z^{b}=3 d \alpha .
$$

Combining these together we obtain

$$
d \alpha=-\frac{2}{3} \iota_{Z} \varphi
$$


Next, consider the identity (11) by taking $\lambda=\varphi, v=Z$ and $\mu=\alpha \wedge(d \alpha)^{2}$ : Using (17), we compute the left-hand side as

$$
\left(\iota_{Z} \varphi\right) \wedge \alpha \wedge(d \alpha)^{2}=-\frac{3}{2} \alpha \wedge(d \alpha)^{3},
$$

and the right-hand side as

$$
\varphi \wedge \iota_{Z}\left(\alpha \wedge(d \alpha)^{2}\right)=\alpha(Z) \varphi \wedge d \alpha \wedge d \alpha=\frac{4}{9}\|Z\|^{2} \varphi \wedge\left(\iota_{Z} \varphi\right) \wedge\left(\iota_{Z} \varphi\right) .
$$

Therefore, by using the identity (2) in the right-hand side, we obtain

$$
\alpha \wedge(d \alpha)^{3}=-\frac{16}{9}\|Z\|^{4} \text { Vol. }
$$

Hence, we conclude that $\alpha \wedge(d \alpha)^{3}$ is a volume form on $M$ (as being a nonzero function multiple of the volume form Vol on $M$ determined by the metric $g_{\varphi}$ ). Equivalently, $\alpha$ is a contact form on $M$. Moreover, it follows from (17) that $\left(1 /\|Z\|^{2}\right) Z$ is the Reeb vector field of $\alpha$, i.e., it satisfies (4). Hence, $\operatorname{Ker}(\alpha)$ is an $A$-compatible contact structure on $(M, \varphi)$.

With the inspiration we get from the proof of Theorem 6.1 , we define a new structure on 7 -manifolds as follows:

Definition 6.2. Let $M^{7}$ be a smooth manifold. A contact- $G_{2}-$ structure on $M$ is a quintuple $(\varphi, R, \alpha, f, g)$ where $\varphi$ is a $G_{2}$-structure, $R$ is a nowhere-zero vector field, $\alpha$ is a 1 -form on $M$, and $f, g: M \rightarrow \mathbb{R}$ are nowhere-zero smooth functions such that

(i) $\alpha(R)=f$

(ii) $d(g \alpha)=\iota_{R} \varphi$

Observe that we have already seen an example of a contact $-G_{2}-$ structure in the above proof (of course under the assumptions of Theorem 6.1) with $R=Z, \alpha=$ $Z^{b}, f=\|Z\|^{2}, g \equiv-3 / 2$. The reason why we call the quintuple $(\varphi, R, \alpha, f, g)$ "contact $-G_{2}-$ structure" is given by the following theorem.

ThEOREM 6.3. Let $(\varphi, R, \alpha, f, g)$ be a contact- $G_{2}-$ structure on a smooth manifold $M^{7}$. Then $\alpha$ is a contact form on $M$. Moreover, $\xi=\operatorname{Ker}(\alpha)$ is an A-compatible contact structure on $(M, \varphi)$. In particular, if $M$ is closed, then it does not admit a contact $-G_{2}-$ structure with $d \varphi=0$.

Proof. We first show that $\alpha$ is a contact form on $M$. Consider the 1-form

$$
\alpha^{\prime}:=g \alpha .
$$

Note that $\operatorname{Ker}(\alpha)=\operatorname{Ker}\left(\alpha^{\prime}\right)$ as $g$ is a nowhere-zero function. Therefore, if we show that $\alpha^{\prime}$ is a contact form on $M$, then it will imply that so is $\alpha$. The conditions in Definition 6.2 translate into

$$
\alpha^{\prime}(R)=f g \text { and } \quad d \alpha^{\prime}=\iota_{R} \varphi .
$$

Also from the equation (2) we get

$$
\left(d \alpha^{\prime}\right)^{2} \wedge \varphi=\left(\iota_{R} \varphi\right) \wedge\left(\iota_{R} \varphi\right) \wedge \varphi=6\|R\|^{2} \text { Vol. }
$$


Now if we write the equation (11) by taking $\lambda=\varphi, \mu=\alpha^{\prime} \wedge\left(d \alpha^{\prime}\right)^{2}$ and $v=R$, then the left-hand side gives

$$
\left(\iota_{R} \varphi\right) \wedge \alpha^{\prime} \wedge\left(d \alpha^{\prime}\right)^{2}=\left(d \alpha^{\prime}\right) \wedge \alpha^{\prime} \wedge\left(d \alpha^{\prime}\right)^{2}=\alpha^{\prime} \wedge\left(d \alpha^{\prime}\right)^{3},
$$

and from the right-hand side we have

$$
\varphi \wedge \iota_{R}\left(\alpha^{\prime} \wedge\left(d \alpha^{\prime}\right)^{2}\right)=\alpha^{\prime}(R) \varphi \wedge\left(d \alpha^{\prime}\right)^{2}=f g \varphi \wedge\left(d \alpha^{\prime}\right)^{2}=6 f g\|R\|^{2} \text { Vol. }
$$

Therefore, we conclude

$$
\alpha^{\prime} \wedge\left(d \alpha^{\prime}\right)^{3}=6 f g\|R\|^{2} \mathrm{Vol}
$$

which implies that $\alpha^{\prime}$ (and so $\alpha$ ) is a contact form on $M$ as $6 f g\|R\|^{2}$ is a nowhere-zero function on $M$.

Next, we consider the vector field $R^{\prime}=(1 / f g) R$. Clearly, $\alpha^{\prime}\left(R^{\prime}\right)=1$. Also we compute

$$
\iota_{R^{\prime}} d \alpha^{\prime}=(1 / f g) \iota_{R} d \alpha^{\prime}=(1 / f g) \iota_{R}\left(\iota_{R} \varphi\right)=0
$$

as $\varphi$ is skew-symmetric. Therefore, $R^{\prime}$ is the Reeb vector field of $\alpha^{\prime}$, and so $\xi=$ $\operatorname{Ker}\left(\alpha^{\prime}\right)$ is an A-compatible contact structure on $(M, \varphi)$ by definition. Finally, the last statement now follows from Theorem 4.3.

The next result shows that we can go also in the reverse direction.

TheOREm 6.4. Let $(M, \varphi)$ be any manifold with $G_{2}$-structure. Then every Acompatible contact structure on $(M, \varphi)$ determines a contact- $G_{2}-$ structure on $M$.

Proof. Let $\xi$ be a given A-compatible contact structure on $(M, \varphi)$. By definition, there exist a non-vanishing vector field $R$ on $M$, a contact form $\alpha$ for $\xi$ and a nowherezero function $h: M \rightarrow \mathbb{R}$ such that $d \alpha=\iota_{R} \varphi$ and $h R$ is the Reeb vector field of some contact form (possibly different than $\alpha$ ) for $\xi$. Being a Reeb vector field, $h R$ is transverse to the contact distribution $\xi$. Therefore, $R$ is also transverse to $\xi$ because $h$ is nowhere-zero on $M$. As a result, there must be a nowhere-zero function $f: M \rightarrow \mathbb{R}$ such that

$$
\alpha(R)=f .
$$

To check this, assume, on the contrary, that the function $M \rightarrow \mathbb{R}$ given by $x \mapsto \alpha_{x}\left(R_{x}\right)$ has a zero, say at $p$. So, we have $\alpha_{p}\left(R_{p}\right)=0$ which means that $R_{p} \in \operatorname{Ker}\left(\alpha_{p}\right)=\xi_{p}$. But this contradicts to the fact that $R$ is everywhere transverse to $\xi$. Hence, we obtain a contact $-G_{2}-$ structure $(\varphi, R, \alpha, f, 1)$. This finishes the proof.

7. Some examples. In this final section, we give some examples of $G_{2}$-manifolds admitting $A$-compatible contact structures. In fact, by Theorem 6.4, in each example we will also have a corresponding contact $-G_{2}-$ structure.

7.1. $C Y \times S^{1}($ or $C Y \times \mathbb{R})$. Consider a well-known example of $G_{2}$-manifold $\left(C Y \times S^{1}, \varphi\right)$ where we assume $C Y(\Omega, \omega)$ is a 3 -fold Calabi-Yau manifold which is either noncompact or compact with boundary. Assume Kähler form $\omega$ on $C Y$ is exact, i.e. $\omega=d \lambda$ for some $\lambda \in \Omega^{1}(C Y)$ and set $\alpha=d t+\lambda$ where $t$ is the coordinate on $S^{1}$. Then $\alpha \wedge(d \alpha)^{3}=\omega^{3} \wedge d t$ is a volume form, and so $\alpha$ is a contact 1-form on $C Y \times S^{1}$. Moreover, $\partial t$ is the Reeb vector field of $\alpha$ as $\iota_{\partial t} \alpha=1$ and $\iota_{\partial t} d \alpha=\iota_{\partial t} \omega=0$. Also observe that since $\varphi=\operatorname{Re}(\Omega)+\omega \wedge d t$ (see [10], for instance), we compute 
$\iota_{\partial t} \varphi=\iota_{\partial t}(\operatorname{Re}(\Omega)+\omega \wedge d t)=\iota_{\partial t} \operatorname{Re}(\Omega)+\iota_{\partial t}(\omega \wedge d t)=\omega \iota_{\partial t} d t=\omega=d \lambda=d \alpha$.

Thus, $\xi=\operatorname{Ker}(\alpha)$ is an A-compatible contact structure on $\left(C Y \times S^{1}, \varphi\right)$, or in other words, $(\varphi, \partial t, \alpha, 1,1)$ is a contact- $G_{2}$-structure on $C Y \times S^{1}$. We note that, by considering $t$ as a coordinate on $\mathbb{R}$, the above argument also gives a contact- $G_{2}$-structure on $C Y \times \mathbb{R}$.

7.2. $W \times S^{1}($ or $W \times \mathbb{R})$. We now give a special case of the above example. First, we need some definitions: A Stein manifold of complex dimension $n$ is a triple $\left(W^{2 n}, J, \psi\right)$ where $J$ is a complex structure on $W$ and $\psi: W \rightarrow \mathbb{R}$ is a smooth map such that the 2 -form $\omega_{\psi}=-d(d \psi \circ J)$ is non-degenerate (and so an exact symplectic form) on $W$. Indeed, $\left(W, J, \omega_{\psi}\right)$ is an exact Kähler manifold. We say that $\left(M^{2 n-1}, \xi\right)$ is Stein fillable if there is a Stein manifold $\left(W^{2 n}, J, \psi\right)$ such that $\psi$ is bounded from below, $M$ is a non-critical level of $\psi$, and $-(d \psi \circ J)$ is a contact form for $\xi$.

Next, consider a parallelizable Stein manifold $(W, J, \psi)$ of complex dimension three. By a result of [6], we know that $c_{1}(W, J)=0$, i.e., the first Chern class of $(W, J)$ vanishes. Therefore, $W$ admits a Calabi-Yau structure with associated Kähler form $\omega_{\psi}=-d(d \psi \circ J)$. Let $\Omega$ be the non-vanishing holomorphic 3-form on $W$ corresponding to this Calabi-Yau structure. Then by the previous example, $\left(W \times S^{1}, \varphi\right)$ is a $G_{2}$-manifold with $\varphi=R e(\Omega)+\omega_{\psi} \wedge d \theta$ (where $\theta$ is the coordinate on $\left.S^{1}\right), \alpha=d \theta-(d \psi \circ J)$ is a contact 1 -form on $W \times S^{1}$ with the Reeb vector field $\partial \theta$, and $\xi=\operatorname{Ker}(\alpha)$ is an A-compatible contact structure on $\left(W \times S^{1}, \varphi\right)$. Again by considering $\theta$ as a coordinate on $\mathbb{R}$, we obtain an A-compatible contact structure on $(W \times \mathbb{R}, \varphi)$. Note that the corresponding contact- $G_{2}$-structure in both cases is $(\varphi, \partial \theta, \alpha, 1,1)$.

Now consider the unit disk $\mathbb{D}^{2} \subset \mathbb{C}$. Then $\left(W \times \mathbb{D}^{2}, J \times i, \psi+|z|^{2}\right)$ is a Stein manifold where $i$ is the usual complex structure and $z=r e^{i \theta}$ is the coordinate on $\mathbb{C}$. Let $\eta$ be the induced contact structure on the boundary

$$
\partial\left(W \times \mathbb{D}^{2}\right)=\left(\partial W \times \mathbb{D}^{2}\right) \cup\left(W \times S^{1}\right) .
$$

Then we remark that the restriction of the Stein fillable structure $\eta$ on $W \times S^{1}$ is the contact structure $\xi$ constructed above.

7.3. $\mathbb{R}^{3} \times K^{4}$. Let $K$ be a Kähler manifold with an exact Kähler form $\omega$, i.e. $\omega=d \lambda$ for some $\lambda \in \Omega^{1}(K)$. Note that $K$ is either noncompact or compact with boundary. Consider the $G_{2}$-manifold $\mathbb{R}^{3} \times K^{4}$ with the $G_{2}$-structure

$$
\varphi=d x_{1} d x_{2} d x_{3}+\omega \wedge d x_{1}+\operatorname{Re}(\Omega) \wedge d x_{2}-\operatorname{Im}(\Omega) \wedge d x_{3}
$$

where $\left(x_{1}, x_{2}, x_{3}\right)$ are the coordinates on $\mathbb{R}^{3}$ (see [10]). Then $\alpha=d x_{1}+x_{2} d x_{3}+\lambda$ is a contact 1-form as $\alpha \wedge(d \alpha)^{3}=d x_{1} d x_{2} d x_{3} \wedge \omega^{2}$ is a volume form on $\mathbb{R}^{3} \times K^{4}$. One can easily check that $\partial x_{1}$ is the Reeb vector field of $\alpha$. Furthermore,

$$
\begin{aligned}
i_{\partial x_{1}} \varphi & =i_{\partial x_{1}}\left(d x_{1} d x_{2} d x_{3}+\omega \wedge d x_{1}+\omega \wedge d x_{2}+\omega \wedge d x_{3}\right) \\
& =d x_{2} d x_{3}+i_{\partial x_{1}}\left(\omega \wedge d x_{1}\right)=d x_{2} d x_{3}+\omega=d\left(x_{2} d x_{3}+\lambda\right)=d \alpha
\end{aligned}
$$

Hence, $\xi=\operatorname{Ker}(\alpha)$ is an A-compatible contact structure on $\left(\mathbb{R}^{3} \times K^{4}, \varphi\right)$ and the corresponding contact- $G_{2}$-structure on $\mathbb{R}^{3} \times K^{4}$ is $\left(\varphi, \partial x_{1}, \alpha, 1,1\right)$.

7.4. $T^{*} M^{3} \times \mathbb{R}$. Let $M$ be any oriented Riemannian 3 -manifold and $T^{*} M$ denote the cotangent bundle of $M$. It is shown in [4] that $T^{*} M \times \mathbb{R}$ has a $G_{2}$-structure $\varphi$ with $d \varphi=0$. To describe $\varphi$, let $\left(x_{1}, x_{2}, x_{3}\right)$ be local coordinates on $M$ around a given point, 
and consider the corresponding standard local coordinates $\left(x_{1}, x_{2}, x_{3}, \xi_{1}, \xi_{2}, \xi_{3}\right)$ on the cotangent bundle $T^{*} M$. These define the standard symplectic structure $\omega=-d \lambda$ on $T^{*} M$ where $\lambda=\Sigma_{i=1}^{3} \xi_{i} d x_{i}$ is the tautological 1-form on $T^{*} M$. Let $t$ denote the coordinate on $\mathbb{R}$. Then $\varphi=R e(\Omega)-\omega \wedge d t$ where $\Omega=\left(d x_{1}+i d \xi_{1}\right) \wedge\left(d x_{2}+i d \xi_{2}\right) \wedge$ $\left(d x_{3}+i d \xi_{3}\right)$ is the complex-valued $(3,0)$-form on $M$. On the other hand, the 1 -form $\alpha=d t+\lambda$ is a contact form on $T^{*} M \times \mathbb{R}$ with the Reeb vector field $\partial t$. Now it is straightforward to check that $\xi=\operatorname{Ker}(\alpha)$ is an $A$-compatible contact structure on $\left(T^{*} M \times \mathbb{R}, \varphi\right)$ and also that $(\varphi, \partial t, \alpha, 1,1)$ is the corresponding contact- $G_{2}$-structure on $T^{*} M \times \mathbb{R}$.

\section{REFERENCES}

[1] D. BlaIR, Contact Manifolds in Riemannian Geometry, Lecture Notes in Mathematics 509 , Springer-Verlag, 1976.

[2] R. L. BRyant, Metrics with exceptional holonomy, Ann. of Math., 126 (1987), pp. 525-576.

[3] R. L. BRyAnt, On the construction of some complete metrics with exceptional holonomy, Duke math. J., 58:3 (1989), pp. 829-850.

[4] H. Cho, S. Salur, And A. J. TOdD, A note on closed G-structures and 3-manifolds, arXiv:1112.0830v1.

[5] H. Cho, S. SAlur, And A. J. TodD, Diffeomorphisms of 7-Manifolds with Closed G $_{2}$-Structure , arXiv:1112.0832v1.

[6] O. Fonster, Some remarks on parallelizable Stein manifolds, Bull. Amer . Math. Soc., 73:5 (1967), pp. $712-716$.

[7] H. Geiges, Contact geometry, Handbook of differential geometry. Vol. II, Elsevier/NorthHolland, Amsterdam, (2006), pp. 315-382.

[8] J. W. GRAY, Some global properties of contact structures, Ann. of Math., 69:2 (1959), pp. 421450.

[9] F. R. Harvey and H. B. Lawson, Calibrated Geometries, Acta. Math., 148 (1982), pp. 47-157.

[10] D. Joyce, The exceptional holonomy groups and calibrated geometry, Proceedings of $11^{\text {th }}$ Gokova Geometry-Topology Conference, (2003), pp. 1-29.

[11] W. Massey, Obstructions to the existence of almost compact structures, Bull. Amer. Math. Soc., 67 (1961), pp. 559-564.

[12] S. SASAKI, On differentiable manifolds with certain structures which are closely related to almost contact structure I, Tôhoku Math. J., 12 (1960), pp. 459-476.

[13] N. Steenrod, The topology of fiber bundles, Princeton Universtiy Press, 1951. 
